\title{
Predictors of injury-related and non-injury-related mortality among veterans with alcohol use disorders
}

\author{
Sylwia Fudalej ${ }^{1,2}$, Amy Bohnert ${ }^{2,3}$, Karen Austin ${ }^{3}$, Kristen Barry ${ }^{2,3}$, Frederic Blow ${ }^{2,3}$ \& \\ Mark IIgen ${ }^{2,3}$
}

Department of Psychiatry, Medical University of Warsaw, Warsaw, Poland,' Department of Psychiatry, University of Michigan, Ann Arbor, MI, USA² and Department of Veterans Affairs National Serious Mental IIIness Treatment Research and Evaluation Center (SMITREC), Ann Arbor, MI, USA ${ }^{3}$

\section{ABSTRACT}

Aims To describe the association between alcohol use disorders (AUDs) and mortality and to examine risk factors for and all-cause, injury-related and non-injury-related mortality among those diagnosed with an AUD. Setting Department of Veterans Affairs, Veterans Health Administration (VHA). Participants A cohort of individuals who received health care in VHA during the fiscal year (FY) 2001 ( $n=3944$ 778), followed from the beginning of FY02 through the end of FY06. Measurements Demographics and medical diagnoses were obtained from VHA records. Data on mortality were obtained from the National Death Index. Findings Controlling for age, gender and race and compared to those without AUDs, individuals with AUDs were more likely to die by all causes [hazard ratio $(\mathrm{HR})=2.30$ ], by injury-related ( $H R=3.29)$ and by non-injury-related causes (HR = 2.21). Patients with AUDs died 15 years earlier than individuals without AUDs on average. Among those with AUDs, Caucasian ethnicity and all mental illness diagnoses that were assessed were associated more strongly with injury-related than non-injury-related mortality. Also among those with AUDs, individuals with medical comorbidity and older age were at higher risk for non-injury related compared to injury-related mortality. Conclusions In users of a large health-care system, a diagnosis of an AUD is associated significantly with increased likelihood of dying by injury and non-injury causes. Patients with a diagnosis of an AUD who die from injury differ significantly from those who die from other medical conditions. Prevention and intervention programs could focus separately upon selected groups with increased risk for injury or non-injury-related death.

Keywords Alcohol use disorders, cohort study, injury-related mortality, non-injury-related mortality.

Correspondence to: Mark Ilgen, Department of Psychiatry, University of Michigan, 4250 Plymouth Road, Ann Arbor, MI 48109, USA.

E-mail: marki@umich.edu

Submitted 28 October 2009; initial review completed 11 December 2009; final version accepted 12 March 2010

\section{INTRODUCTION}

Approximately 76 million individuals have a diagnosable alcohol use disorder (AUD) world-wide [1]. Heavy use of alcohol and/or AUDs have been linked with more than 60 types of injury and disease [2,3]. The World Health Organization (WHO) estimates that alcohol causes 1.8 million deaths globally, which is $3.2 \%$ of all deaths [2]. Alcohol use is a leading preventable cause of death in the United States, with 85000 alcohol-attributable deaths in the United States annually [4].

Almost half of alcohol-related deaths internationally are due to injury; $32 \%$ of deaths attributable to alcohol are from unintentional injuries (including road traffic injuries, drowning, burns, poisoning and falls) and about
$14 \%$ from intentional injuries such as suicide or homicide [5]. Similarly, alcohol is involved in $20-30 \%$ of all fatal and non-fatal injuries [6-8] and unintentional injuries account for $28 \%$ of the disability-adjusted life years attributable to alcohol [9-11]. In the United States, reducing deaths and injuries caused by alcohol-related motor vehicle crashes and alcohol-related violence were identified to be among the most significant preventable threats to the national health [12]. In order to understand more clearly and develop prevention strategies to address alcohol-related deaths, more work is needed to identify specific risk factors for both injury-related and non-injury-related mortality in those with AUDs.

Although only limited longitudinal research has examined risk factors for injury-related mortality among 
individuals with AUDs, longitudinal predictors of allcause mortality in those with an AUD have been studied in samples of alcohol treatment-seeking individuals. This work has generally identified those with heavier use of alcohol, fewer social resources, greater psychiatric and medical comorbidity and older age to be at greater risk of mortality [13-17]. This research indicates that, on average, those with AUDs die 15 years earlier than those without AUDs [16,18]. However, the vast majority of those with AUDs never receive formal alcohol-related treatment and the risk factors for mortality in these individuals are poorly understood. Additionally, prior studies examined mortality by all causes and have not examined differences in risk factors for injury-related and noninjury-related mortality in a single sample. Those who die from injury are likely to differ from those who die from non-injury-related causes and, consequently, it is important to examine these outcomes separately.

Prior research has examined characteristics of those who are injured (either fatally or non-fatally) while intoxicated [19-24]. This research has found generally that those with alcohol-related injuries tended to be male, younger, with lower levels of education and a greater likelihood of reporting violence-related injury when compared to those with non-alcohol-related injuries [24]. Among alcohol-dependent individuals, those who exhibit more episodes of drinking are more often exposed to risk of injury and have a greater risk for injury over time [25]. Although this research provides information about individuals with alcohol-related emergency visits, this work is primarily cross-sectional and the longitudinal predictors of injury (particularly fatal injury) are not wellstudied.

There are few national health care organizations in the United States that have developed and maintain largescale data monitoring systems encompassing facilities and patients across the country. The Veterans Health Administration (VHA), one of the largest health-care systems in the United States, has a medical monitoring system that is a longitudinal resource to understand mental and physical health diagnoses, morbidity and mortality, and health-care utilization in the VHA nationally. This present study focused upon the population of all individuals diagnosed with an AUD by any provider in the Department of Veterans Affairs (VA) health-care system within a single year. Veterans are significantly more likely to suffer from alcohol dependence than their civilian counterparts [26,27], with a little over $20 \%$ of VA users meeting criteria for hazardous drinking [28].

The purpose of this descriptive study was to: (i) describe the overall association between a diagnosis of an AUD and all-cause mortality, non-injury-related and injury-related mortality within 5 years of receiving care in the VA; and (ii) examine the demographic and clinical characteristics of patients with AUDs as longitudinal predictors of non-injury-related and injury-related mortality over the course of 5 years. We further examined differences between the two outcomes (injury mortality and non-injury mortality) in their associations with particular patient characteristics using a competing risks approach.

\section{MATERIALS AND METHODS}

All study data were obtained from the VA's National Patient Care Database (NPCD) and linked to mortality data from the CDC's National Death Index (NDI) Plus database. The NPCD includes records of all clinical visits in the VA system, as well as information on patient demographics and medical and psychiatric diagnoses. The study was approved by the Institutional Review Board at the Ann Arbor VA.

The entire population of individuals who utilized VA services in fiscal year 2001 (FY01) (1 October 2000-30 September 2001; $n=3944778$ ) were identified. We first examined the general association between AUD diagnosis and mortality (both injury- and non-injury-related) in this overall sample. All subsequent analyses were conducted on the subset of 122427 individuals who were diagnosed with an AUD during FY01. The time of observation used to analyse mortality among individuals who used services in FY01 started at the beginning of FY02 and extended to the end of FY06.

Measures of vital status and cause of death were based on information from the National Death Index (NDI). The data obtained from NDI included dates and causes of death for all US residents who die within the United States (excluding New York City), derived from death certificates filed in state vital statistics offices. NDI searches were performed for VA patients who received any VA service use during FY01, and who had no subsequent VA service use through the end of FY06. This design used VA service encounters subsequent to the study observation period as an indicator of having survived the observation period.

The NDI data request included Social Security Number, last name, first name, middle initial, date of birth, race/ethnicity, sex and state of residence. NDI search results often include multiple records that are potential matches. 'True matches' were identified based on established procedures [29]. More than $99 \%$ of deaths in the VHA user population since FY99 have matched on all nine digits of the Social Security Number in NDI records.

To identify injury-related deaths, we used the ICD-10 injury mortality framework which was developed to be as consistently as possible with the CDC-recommended framework developed for the ICD-9 external cause of 
injury codes [29]. The cause of death was coded using 16 categories developed by the National Center for Health Statistics [30]. For the present study, these 16 categories were reduced to two categories: injury-related (including: suicide, accidents and homicide) and non-injury-related (including: heart disease, malignant neoplasms, cerebrovascular diseases, chronic lower respiratory diseases, diabetes, Alzheimer's disease, influenza plus pneumonia, nephritis, septicemia, liver disease or damage, hypertension, Parkinson's disease and other cause).

Demographic (age, gender and ethnicity, which was categorized into Caucasian, African American or other) and clinical characteristics (medical comorbidity, psychiatric comorbidities) were examined as longitudinal predictors of injury-related and non-injury-related mortality. Medical comorbidities were examined using the Charlston Comorbidity Index for administrative data [31,32], which classifies comorbidity by severity. Psychiatric diagnoses included schizophrenia, bipolar disorder, major depression, personality disorders, post-traumatic stress disorder (PTSD), other anxiety disorders and drug use disorders.

\section{Analyses}

We first examined the association between an AUD diagnosis and risk of all-cause mortality, injury-related and non-injury-related mortality in the population of FY01 VA patients using Cox's proportional hazard regression. Because of the strong association between age, gender and the risk of mortality, we examined the association between an AUD and mortality controlling for age (as a continuous covariate), race and gender and then examined the relationship stratified by age group (18-44, 45-64 and 65+ years). We next calculated mean age at death by AUD status.

The remaining analyses focused upon those who were diagnosed with an AUD. We calculated $\chi^{2}$ tests of the association between demographic, medical and clinical characteristics with a three-category mortality outcome (alive, injury-related mortality and non-injury-related mortality) among those individuals with an AUD. We conducted multivariable competing risks Cox's proportional hazards regression analysis to examine predictors of injury and non-injury mortality simultaneously, using the Lunn-McNeil approach [33]. This method allowed us to estimate the hazard ratios (HRs) for each covariate for injury-related and non-injury-related deaths separately, and to test if the estimated HRs for each covariate differed significantly between the two types of fatality outcomes (using method B, described in [33]). SAS version 9.1 was used for all analyses. The results were considered statistically significant at a level of $P<0.05$.

\section{RESULTS}

During follow-up, 661317 of the 3944778 VA service users in FY01 died (16.8\%). Among the subsample of individuals with an AUD $(n=122$ 427), 21926 died (17.9\%). Among the full population of individuals who used VA services in FY01, individuals with AUDs had a 2.30 times greater hazard of dying by all-cause, 3.29 times greater hazard of dying by injury and 2.21 times greater hazard of dying by non-injury compared to those with AUDs after controlling for age, race and gender (Table 1). Similarly, mortality by all causes was more common in those individuals with AUDs than those without in each age group analyzed, controlling for gender and race (all $P<0.001$ ). The risk of all-cause mortality for those with AUDs relative to those without was greatest for individuals in the youngest age group ( $\mathrm{HR}=2.68,95 \% \mathrm{CI}=2.55-2.81)$. AUD diagnosis was also a significant predictor of injury-related mortality in all age groups $(P<0.001$ in each age group analyzed). Participants with AUDs under the age of 45 had 3.14 times [95\% confidence interval $(\mathrm{CI})=2.88-3.42]$ greater hazard of injury-related death than individuals without AUDs in this age group. A diagnosis of an AUD also increased the risk of non-injury-related mortality significantly (all $P<0.001$ ). The strongest association of AUDs

Table 1 Cox's proportional hazards model for effect of alcohol use disorders (AUDs) on time to mortality during fiscal years 2002 (FY02)-FY06 ( $n=3944778)$.

\begin{tabular}{|c|c|c|c|c|c|c|c|}
\hline & \multirow[b]{2}{*}{$n(\%)$} & \multicolumn{2}{|c|}{ All-cause mortality } & \multicolumn{2}{|c|}{ Injury-related } & \multicolumn{2}{|c|}{ Non -injury-related } \\
\hline & & $H R$ & $95 \% C I$ & $H R$ & $95 \% C I$ & $H R$ & $95 \% C I$ \\
\hline Adjusting for age, race and gender & $3944778(100 \%)$ & 2.30 & $(2.27,2.33)^{* *}$ & 3.29 & $(3.15,3.44)^{* *}$ & 2.21 & $(2.17,2.24)^{* *}$ \\
\hline \multicolumn{8}{|c|}{ By age group, adjusted for race and gender } \\
\hline$<45$ years & $598528(15.2)$ & 2.68 & $(2.55,2.81)^{* *}$ & 3.14 & $(2.88,3.42)^{* *}$ & 2.50 & $(2.36,2.65)^{* *}$ \\
\hline $45-64$ years & $1517434(38.5)$ & 1.99 & $(1.96,2.03)^{* *}$ & 3.22 & $(3.05,3.39)^{* *}$ & 1.90 & $(1.86,1.93)^{* *}$ \\
\hline 65 years or older & $1828816(46.4)$ & 1.57 & $(1.53,1.61)^{* *}$ & 2.05 & $(1.77,2.437)^{* *}$ & 1.56 & $(1.51,1.60)^{* *}$ \\
\hline
\end{tabular}

${ }^{* *} \mathrm{P}<0.001$. CI: confidence interval; HR: hazard ratio. 
Table 2 Cause of death in those with alcohol use disorders (AUDs) ( $n=21926$ deaths).

\begin{tabular}{lrrr}
\hline Disease categories & Frequency & $\%$ & Cumulative \% \\
\hline Heart disease & 4710 & 21.5 & 21.5 \\
Malignant neoplasms & 4349 & 19.8 & 41.3 \\
Liver disease or damage & 2086 & 9.5 & 50.8 \\
Accidents* & 1789 & 8.2 & 59 \\
Chronic lower respiratory & 1144 & 5.2 & 64.2 \\
$\quad$ diseases & & & \\
Cerebrovascular diseases & 549 & 2.5 & 66.7 \\
Suicide* & 551 & 2.5 & 69.2 \\
Diabetes & 435 & 2.1 & 71.3 \\
Influenza and pneumonia & 347 & 1.6 & 72.9 \\
Septicemia & 333 & 1.5 & 74.4 \\
Nephritis & 235 & 1.1 & 75.5 \\
Homicide* & 205 & 0.9 & 76.4 \\
Hypertension & 134 & 0.6 & 77 \\
Alzheimer's disease & 73 & 0.3 & 77.3 \\
Parkinson's disease & 27 & 0.1 & 77.4 \\
Other medical conditions & 4959 & 22.6 & 100 \\
& & & \\
\hline
\end{tabular}

*Injury-related causes of mortality.

with non-injury-related mortality was found in the youngest age group ( $\mathrm{HR}=2.50,95 \% \mathrm{CI}=2.36-2.65)$. Furthermore, the increase in odds of both injury and noninjury-related mortality for those with an AUD compared to those without was greater in the 45-65 age group than the over- 65 age group. Those with AUDs died at an earlier age than those without AUDs [59.7 (standard deviation, $\mathrm{SD}=10.9)$ versus $75.2(\mathrm{SD}=10.7)]$. Those with AUDs died earlier of both injury-related causes [52.3 (SD = 9.5) versus $66.9(\mathrm{SD}=15.5)$ ] and non-injury-related causes [60.7 $(\mathrm{SD}=10.7)$ versus $75.5(\mathrm{SD}=10.4)]$.

The remaining analyses focus upon those individuals with AUDs. Table 2 presents the specific causes of death in those with AUDs in the order of most to least frequent specific cause of death (with 'other medical conditions' listed last). The most common causes of death among patients with AUDs were heart disease (21.5\%) and malignant neoplasms (19.8\%). Injury-related mortality accounted for $11.6 \%$ of all mortality in those with AUDs. More than $70 \%(70.3 \%)$ of injury-related deaths were due to accidents, $21.7 \%$ to suicide and $8.1 \%$ to assault.

Table 3 provides an overall description of those who were alive at the end of observation, those who died by injury, and those who died by non-injury causes among those with AUDs. Additionally, bivariate tests of the association between patient characteristics and a threecategory outcome variable (alive, died by injury, died by non-injury) are presented in Table 3. These results indicate differences between groups among VA patients with AUDs for all characteristics examined $(P<0.001)$, including age, ethnicity, gender, the presence of medical comorbidity, drug use disorders, personality disorders and other psychiatric comorbidity (PTSD, major depression, bipolar disorder, schizophrenia, and non-PTSD anxiety disorders).

In our next steps of modeling, we sought to extend our bivariate analysis by both controlling for demographic and clinical characteristics and determining between which of the three possible outcomes (alive, injury death, non-injury death) significant differences lie. A multivariable competing-risks Cox's proportional hazards regression model was used with the same three-category outcome variable as the one used in the previous step of analysis and the 'alive' group as the reference. The following predictors increased the likelihood of injury-related death compared to being alive at the end of the observation period among individuals with AUDs (see Table 4): Caucasian ethnicity (compared to black), medical comorbidity, personality disorders, drug use disorders and nonPTSD anxiety disorders. Female gender and older age decreased the likelihood of injury-related death. Predictors of lower likelihood of non-injury-related death compared to being alive at the end of the observation period among individuals with AUDs were: female gender, schizophrenia, PTSD, other anxiety disorders, bipolar disorder, personality disorders, major depression and drug use disorders. Medical comorbidity, older age and Caucasian ethnicity increased the likelihood of non-injuryrelated mortality compared to being alive at the end of the observation period among individuals with AUDs.

An additional competing-risks Cox's proportional hazards regression model using interaction terms to test the equivalence of the estimated effects of each covariate on the two mortality outcomes revealed that a number of patient characteristics were more common among those who died of injury-related causes than those who died of non-injury-related causes among VA users with AUDs. These included: Caucasian ethnicity and a diagnosis of schizophrenia, PTSD, other anxiety disorders, bipolar disorder, personality disorders, major depression and drug use disorders. Comparisons also indicated that medical comorbidity and older age were more common among those who died of injury causes than those who died of non-injury causes.

\section{DISCUSSION}

This large-scale study of all individuals who used VA services within a single year confirmed that a diagnosis of an AUD increased mortality risk significantly. To our knowledge, this is the only study to examine these associations in the full population of users of a single health system. Much of the prior research relied on samples of addictions treatment-seeking individuals: presumably, those with more severe AUDs. On average, patients with an 
Table 3 Patient characteristics and mortality in those with alcohol use disorders (AUDs) $(n=122427)$.

\begin{tabular}{|c|c|c|c|c|}
\hline Predictor & Alive & $\begin{array}{l}\text { Non-injury-related } \\
\text { death }\end{array}$ & $\begin{array}{l}\text { Injury-related } \\
\text { death }\end{array}$ & $\chi^{2}$ \\
\hline \multirow[t]{2}{*}{ Total } & 100501 & 19381 & 2545 & \\
\hline & $\%$ & $\%$ & $\%$ & \\
\hline Age (years) & & & & $9441.93^{* *}$ \\
\hline$<45$ & 34.04 & 9.38 & 31.94 & \\
\hline $45-64$ & 59.70 & 65.90 & 61.61 & \\
\hline$\geq 65$ & 6.26 & 24.72 & 6.44 & \\
\hline Race & & & & $1607.39^{* *}$ \\
\hline Black & 25.79 & 19.83 & 15.64 & \\
\hline White & 43.08 & 57.90 & 57.05 & \\
\hline Other & 31.13 & 22.27 & 27.31 & \\
\hline Gender & & & & $205.88^{* *}$ \\
\hline Female & 3.09 & 1.27 & 1.93 & \\
\hline Male & 96.91 & 98.73 & 98.07 & \\
\hline Medical comorbidity & & & & $10127.49^{* *}$ \\
\hline None & 59.94 & 27.90 & 49.35 & \\
\hline 1 & 26.00 & 29.74 & 28.13 & \\
\hline 2 & 14.06 & 42.37 & 22.51 & \\
\hline Drug use disorder & & & & $1345.79^{* *}$ \\
\hline No & 42.73 & 56.80 & 38.27 & \\
\hline Yes & 57.27 & 43.20 & 61.73 & \\
\hline Schizophrenia & & & & $139.27^{* *}$ \\
\hline No & 89.29 & 92.02 & 87.94 & \\
\hline Yes & 10.71 & 7.98 & 12.06 & \\
\hline PTSD & & & & $153.09^{* *}$ \\
\hline No & 82.87 & 86.23 & 80.04 & \\
\hline Yes & 17.13 & 13.77 & 19.96 & \\
\hline Other anxiety disorder & & & & $79.74^{* *}$ \\
\hline No & 91.23 & 92.43 & 87.43 & \\
\hline Yes & 8.77 & 7.57 & 12.57 & \\
\hline Bipolar disorder & & & & $238.65^{* *}$ \\
\hline No & 89.90 & 93.17 & 86.72 & \\
\hline Yes & 10.10 & 6.83 & 13.28 & \\
\hline Personality disorder & & & & $298.66^{* *}$ \\
\hline No & 91.39 & 94.69 & 87.54 & \\
\hline Yes & 8.61 & 5.31 & 12.46 & \\
\hline Major depression & & & & $199.85^{* *}$ \\
\hline No & 84.85 & 88.56 & 82.20 & \\
\hline Yes & 15.15 & 11.44 & 17.80 & \\
\hline
\end{tabular}

${ }^{* *} P<0.001$. PTSD: post-traumatic stress disorder.

AUD diagnosis died 15 years earlier than individuals without an alcohol-related diagnosis. This is consistent with previous research $[16,18]$ and highlights the substantial loss of life associated with AUDs. We found that individuals with AUDs had a greater increase in risk for injury-related fatality $(\mathrm{HR}=3.3)$ than non-injuryrelated fatality $(\mathrm{HR}=2.2$ ) relative to individuals without an AUD. However, we also found that nearly 90\% of deaths among individuals with AUDs in this population were attributed to non-injury causes. Collectively, this suggests that while individuals with AUDs have a notably high risk of fatal injury, the role of alcohol in the development of non-trauma medical conditions has larger public health significance than injury-related death.
The risks of all-cause mortality, injury-related and non-injury-related mortality among those with AUDs were higher than among those without AUDs in each age group. Interestingly, the increase in the risk of mortality for individuals with an AUD appears to attenuate with older age, although there was still a statistically significant increase in mortality risk in the oldest age group. Individuals with AUDs under 45 years of age were at almost 2.7 times higher risk of death by all-cause, 3.1 times higher risk of injury-related death and 2.5 times higher risk of non-injury-related death than participants in the same age group without a diagnosis of AUD. This finding is consistent with previous research which found that alcoholic men in the group aged 35-44 years have 
Table 4 Predictors of injury-related versus non-injury-related death among those with alcohol use disorders (AUDs) in a multivariable competing risks Cox's proportional hazards regression model $(n=122427)$.

\begin{tabular}{|c|c|c|c|c|}
\hline \multirow[b]{3}{*}{ Predictors } & \multicolumn{2}{|c|}{ Injury-related death } & \multicolumn{2}{|c|}{ Non-injury-related death } \\
\hline & \multicolumn{2}{|c|}{$n=2545$} & \multicolumn{2}{|c|}{$n=19381$} \\
\hline & $H R$ & $95 \% C I$ & $H R$ & $95 \% C I$ \\
\hline Age+ & 0.991 & $0.987-0.996^{*}$ & 1.053 & $1.051-1.054^{*}$ \\
\hline Caucasian ethnicity $\dagger$ & 2.16 & $1.93-2.42^{*}$ & 1.32 & $1.28-1.38^{*}$ \\
\hline Other ethnicity $\dagger$ & 1.59 & $1.40-1.80^{*}$ & 0.97 & $0.92-1.01$ \\
\hline Female gender & 0.58 & $0.43-0.77^{*}$ & 0.73 & $0.65-0.83^{*}$ \\
\hline Medical comorbidity $\dagger$ & 1.33 & $1.23-1.45^{*}$ & 2.60 & $2.52-2.69^{*}$ \\
\hline Schizophrenia $\dagger$ & 1.08 & $0.96-1.22$ & 0.90 & $0.86-0.95^{*}$ \\
\hline PTSD $\dagger$ & 1.07 & $0.97-1.18$ & 0.86 & $0.82-0.89^{*}$ \\
\hline Other anxiety disorders $\dagger$ & 1.24 & $1.10-1.40^{*}$ & 0.93 & $0.88-0.99^{*}$ \\
\hline Bipolar disorder $\dagger$ & 1.10 & $0.98-1.25$ & 0.85 & $0.81-0.90^{*}$ \\
\hline Personality disorders $\dagger$ & 1.28 & $1.13-1.45^{*}$ & 0.87 & $0.82-0.93^{*}$ \\
\hline Major depression $\dagger$ & 1.02 & $0.92-1.14$ & 0.86 & $0.82-0.90^{*}$ \\
\hline Drug use disorders $\dagger$ & 1.28 & $1.18-1.39^{*}$ & 0.88 & $0.85-0.91^{*}$ \\
\hline
\end{tabular}

${ }^{*} P<0.05$; indicates significant difference between those who died (by injury or non-injury causes, depending on column) and those who were alive. $\dagger P<0.05$; indicates significant difference in the hazard ratios (HR) estimated for injury-related and non-injury-related death, which was determined by a competing risks model with interaction terms (Lunn \& McNeal [33], method B). CI: confidence interval; PTSD: post-traumatic stress disorder.

notably high rates of premature death [16]. It is also consistent with studies that have found that younger individuals with AUDs had a greater increase in mortality than older individuals with AUDs when both were compared with non-alcohol-abusing, age-matched control groups $[14,15,34]$.

The prevalence and predictors of all-cause mortality in individuals with AUDs have been studied previously in samples of alcohol treatment-seeking individuals. This work has found that white ethnicity, male gender, more severe course of alcohol abuse, fewer social resources, greater psychiatric and medical comorbidity and older age increase risk of mortality among those with AUDs [13-17,35]. Our results were generally consistent with this work.

Although prior work has repeatedly described the strong link between alcohol and injury in patients presenting for emergency care [10,24,25], the unique relationship between a diagnosis of an AUD and injuryrelated mortality has received little attention in the literature. The present findings indicate that those who died due to injury were younger and more likely to be male, Caucasian, to have drug use disorders and to have medical comorbidity than those who were alive at the end of observation. Prior research [19-24] has also found that those with alcohol-related injuries are more likely to be male, younger, to use drugs, to use alcohol frequently (except for alcohol use 'almost daily') and to report larger quantities of alcohol consumed than those with nonalcohol-related injuries. Despite the differences in design between the prior research and the present study, the findings are quite similar. We can hypothesize that many of the characteristics identified in the study and prior research (e.g. younger age, male gender, greater severity of substance use) are associated with more impulsive and risky behavior which, in turn, increase the probability of injury and injury-related mortality. Taken together, this work could inform the development of simple measures to assess injury risk that could be administered in AUD treatment settings to identify individuals appropriate for injury-reduction interventions.

The present examination of predictors of both injuryrelated and non-injury-related mortality simultaneously represents a novel contribution to the literature. In the present study, some of the previously examined risk factors were associated with both injury-related and noninjury-related mortality, while others were unique risk or protective factors for one or the other. The results indicate that Caucasian ethnicity, medical comorbidity and male gender were risk factors for both types of mortality. Personality disorders, other anxiety disorders and drug use disorders were found to be associated with increased risk only for injury-related death. These three disorders were associated with lower risk of non-injury-related mortality. Thus, examining only all-cause mortality would have prevented our ability to detect the effect of personality, drug use and anxiety disorders on an important subset of deaths in those with AUDs.

When examining injury and non-injury-related mortality separately, we also found several unexpected findings. Specifically, all the psychiatric disorders were associated with slightly lower risk of non-injury-related 
mortality. Although the magnitude of this association was modest (HRs ranging from 0.85 to 0.93 ) the pattern of findings was consistent. These findings may represent a survival bias. This explanation is most consistent with the findings related to personality disorders, other anxiety disorders and drug use disorders because individuals with these conditions were both more likely to die by injury and less likely to die from non-injury causes. The findings for other psychiatric conditions fitted this overall pattern, although the association between these conditions and injury-related mortality did not reach significance. Subsequent analyses indicated that among those who died, all psychiatric conditions were associated with a greater risk of injury-related mortality compared to non-injury-related mortality. It is possible that Veterans with AUDs and co-occurring psychiatric conditions receive more extensive care than those with only AUDs, and this may reduce their likelihood of mortality due to illness.

The present study should be interpreted with caution for several reasons. All analyses were undertaken for the purpose of hypothesis generation and the extent to which specific risk factors caused mortality directly is unknown. The study was based on individuals who use the VA health-care system and the results may not generalize to the general population of the United States or other countries. Because the vast majority of individuals in this study were male, this limitation may be particularly salient when generalizing the findings to women. The reliability and validity of the measures of mortality are limited by the completeness and accuracy of the data contained within the NDI. The NDI does not include deaths that occurred outside the United States. All diagnoses were provided during medical care (primary and psychiatric) and may differ from diagnoses found with structured diagnostic interviews. Information about drinking patterns and blood alcohol concentration at the time of death, which could enrich study findings, was not available. Although it is not possible to compare directly the results of the bivariate analyses to those of the multivariable analyses, it is notable that several of the associations between risk factors and different forms of mortality appear to be weaker in the multivariable models. This could be due partially to the fact that the impact of some risk factors on mortality (e.g. schizophrenia) are accounted for more effectively by other demographic or diagnostic factors.

This nation-wide study provides new data on the predictors of injury-related and non-injury-related mortality among individuals with AUDs. The present study identifies important differences in risk factors for these two types of mortality and provides detailed information about mortality risk in the population of individuals with an AUD. This study indicates that patients with a diagnosis of an AUD who die from injury differ significantly from those who die from other medical conditions. When examining mortality in those with AUDs, these outcomes should be analyzed separately. Depending on the outcome of interest, prevention and intervention programs could focus separately upon selected target groups with increased risk for injury or non-injury-related death.

\section{Declarations of interest}

The authors have no financial or other interests that might lead to a conflict of interest. None of the authors have connections with the tobacco, alcohol, pharmaceutical and gaming industries or any contractual constraints on publishing with regard to the research being reported.

\section{Acknowledgements}

The authors wish to acknowledge the National Institutes of Health Fogarty International Collaborative Injury and Alcohol Training Program and VHA's Office of Mental Health Services. The study was supported by the Fogarty International Center/NIAAA International Collaborative Alcohol and Injury Research Training Program (5D43TW007569-04) and funds from the Department of Veterans Affairs.

\section{References}

1. World Health Organization. Global status report on alcohol. 2004. Available at: http://www.who.int/substance_abuse/ publications/global_status_report_2004_overview.pdf (accessed 1 July 2009).

2. World Health Organization. The world health report. Reducing Risks, Promoting Healthy Life. 2002. Available at: http://www.who.int/whr/2002/en/whr02_en.pdf (accessed 1 July 2009).

3. Room R., Graham K., Rehm J., Jernigan D., Monteiro M. Drinking and its burden in a global perspective: policy considerations and options. Eur Addict Res 2003; 9: 165-75.

4. Mokdad A. H., Marks J. S., Stroup D. F., Gerberding J. L. Actual causes of death in the United States, 2000. JAMA 2004; 291: 1238-45.

5. World Health Organization. 2009 Alcohol and Injury in Emergency Departments. http://www.who.int/substance_ abuse/publications/alcohol_injury_summary.pdf (accessed 1 July 2009).

6. Honkanen R. Alcohol in home and leisure injuries. Addiction 1993; 88: 939-44.

7. Mahadevan S., Hawton K., Casey D. Deliberate self-harm in Oxford University students, 1993-2005: a descriptive and case-control study. Soc Psychiatry Psychiatr Epidemiol 2010; 45: 211-9.

8. Smith G. S., Branas C. C., Miller T. R. Fatal nontraffic injuries involving alcohol: a metaanalysis. Ann Emerg Med 1999; 33: 659-68.

9. Rehm J., Gmel G., Sempos C. T., Trevisan M. Alcohol-related morbidity and mortality. Alcohol Res Health 2003; 27: 39-51.

10. Cherpitel C. J., Ye Y., Bond J., Rehm J., Poznyak V., 
Macdonald S. et al. Multi-level analysis of alcohol-related injury among emergency department patients: a crossnational study. Addiction 2005; 100: 1840-50.

11. Ezzati M., Lopez A. D., Rodgers A., Vander Hoorn S., Murray C. J. L. Selected major risk factors and global and regional burden of disease. Lancet 2002; 360: 1347-60.

12. Office of Disease Prevention and Health Promotion. Healthy people. 2010. Available at: http://www.healthypeople.gov/ (accessed 1 July 2009).

13. Finney J. W., Moos R. H. The long-term course of treated alcoholism: II. Predictors and correlates of 10-year functioning and mortality. J Studies Alcohol 1992; 53: 142-53.

14. Moos R. H., Brennan P. L., Mertens J. R. Mortality rates and predictors of mortality among late-middle-aged and older substance abuse patients. Alcohol Clin Exp Res 1994; 18: 187-95.

15. Feuerlein W., Küfner H., Flohrschütz T. Mortality in alcoholic patients given inpatient treatment. Addiction 1994; 89: 841-9.

16. Liskow B. I., Powell B. J., Penick E. C., Nickel E. J., Wallace D., Landon J. F. et al. Mortality in male alcoholics after ten to fourteen years. J Studies Alcohol 2000; 61: 853-61.

17. Johnson J. E., Finney J. W., Moos R. H. Predictors of 5-year mortality following inpatient/residential group treatment for substance use disorders. Addict Behav 2005; 30: 130016 .

18. Ojesjö L., Hagnell O., Otterbeck L. Mortality in alcoholism among men in the Lundby Community Cohort, Sweden: a forty-year follow-up. J Studies Alcohol 1998; 59: 140-5.

19. Selway J. S., Soderstrom C. A., Kufera J. A. Alcohol use and testing among older trauma victims in Maryland. J Trauma 2008; 65: 442-6.

20. National Centre for Statistics and Analysis Traffic Safety facts. National Centre for Statistics and Analysis of the National Highway Traffic Safety Administration. 2003. http://www-nrd.nhtsa.dot.gov/Pubs/809822.PDF (accessed 1 July 2009).

21. Heng K., Hargarten S., Layde P., Craven A., Zhu S. Moderate alcohol intake and motor vehicle crashes: the conflict between health advantage and at-risk use. Alcohol Alcohol 2006; 41: 451-4.

22. Reynaud M., Le Breton P., Gilot B., Vervialle F., Falissard B. Alcohol is the main factor in excess traffic accident fatalities in France. Alcohol Clin Exp Res 2002; 26: 1833-9.

23. Touquet R., Csipke E., Holloway P., Brown A., Patel T., Seddon A. J. et al. Resuscitation room blood alcohol concentrations: one-year cohort study. Emerg Med J 2008; 25: 752-6.

24. Borges G., Cherpitel C. J., Orozco R., Bond J., Ye Y., Macdonald S. et al. Acute alcohol use and the risk of non-fatal injury in sixteen countries. Addiction 2006; 101: 993-1002.

25. Borges G., Cherpitel C. J., Medina-Mora M. E., Mondragón L., Casanova L. Alcohol consumption in emergency room patients and the general population: a population-based study. Alcohol Clin Exp Res 1998; 22: 1986-91.

26. Kulka R. A., Schlenger W. E., Fairbank J. A., Hough R. L., Jordan B. K., Marmar C. R. et al. Trauma and the Vietnam War Generation: Report of Findings from the National Vietnam Veterans Readjustment Study. New York: Brunner/Mazel; 1990.

27. Department of Defense. Health Related Behaviors Survey. 2009. Available at: http://www.tricare.osd.mil/main/ news/DoDSurvey.htm\#tab3 (accessed 1 July 2009).

28. Bradley K. A., Williams E. C., Achtmeyer C. E., Volpp B., Collins B. J., Kivlahan D. R. Implementation of evidencebased alcohol screening in the Veterans Health Administration. Am J Manage Care 2006; 12: 597-606.

29. Centers for Disease Control and Prevention. Recommended framework for presenting injury mortality data. Morb Mort Wkly Rep 1997; 46: 1-30.

30. National Centre for Health Statistics. FastStat Homepage: Deaths and Mortality. 2009. Available at. http://www. cdc.gov/nchs/FASTATS/deaths.htm (accessed 1 July 2009).

31. Quan H., Sundararajan V., Halfon P., Fong A., Burnand B., Luthi J. C. et al. Coding algorithms for defining comorbidities in ICD-9-CM and ICD-10 administrative data. Med Care 2005; 43: 1130-9.

32. Charlson M. E., Pompei P., Ales K. L., Mackenzie C. R. A new method of classifying prognostic comorbidity in longitudinal studies: development and validation. J Chronic Dis 1987; 40: $373-83$.

33. Lunn M., Mcneil D. Applying Cox regression to competing risks. Biometrics 1995; 51: 524-32.

34. Yoshino A., Kato M., Yoshimasu H., Tatsuzawa Y., Watanabe T. Which relapse criteria best predict the mortality risk of treated alcoholics? Alcohol Clin Exp Res 1997; 21: 1374-8.

35. Timko C., Debenedetti A., Moos B., Moos R. Predictors of 16-year mortality among individuals initiating helpseeking for an alcoholic use disorder. Alcohol Clin Exp Res 2006; 30: 1711-20. 